\title{
Reaction of electric and meteorological states of the near-ground atmosphere during a geomagnetic storm on 5 April 2010
}

\author{
Sergey Smirnov
}

\begin{abstract}
The effects of a geomagnetic storm on 5 April 2010 on electric parameters of the atmospheric near-ground layer in Kamchatka have been investigated. Three processes over the course of the storm were identified. Air electroconductivity began to decrease $4 \mathrm{~h}$ before the storm, and this lasted for $20 \mathrm{~h}$. The storm's sudden commencement caused potential gradient oscillations with amplitudes up to $300 \mathrm{~V} / \mathrm{m}$. During the stages of the storm, a significant increase in the atmosphere ion content unipolarity coefficient occurred.
\end{abstract}

\section{Findings Introduction}

Solar activity induced geomagnetic storms, and the resulting changes in Earth's atmospheric electricity at high and mid-latitudes have been investigated in many papers (e.g., Apsen et al. 1988). Unfortunately, these results are very limited and are often contradictory. The latter fact may be associated not only with the peculiarities of physical processes in the near-ground atmosphere for separate geomagnetic storms but also with the choice of registration location, for example, as well as the atmospheric state before and during a storm and so on. Earlier, the author of the paper investigated the effects of weak (Mikhailova et al. 2009) and extreme (Smirnov et al. 2013) geomagnetic storms on electric field intensity variations and meteorological value variations during an extreme storm in November 2004.

The electric field in the atmosphere shows the Earthionosphere potential difference. In the paper by Markson (1981), a positive correlation between the lower ionosphere electric potential and the galactic cosmic ray (GCR) intensity was determined. In other experiments in a mountainous country, a negative correlation of these parameters was discovered. Furthermore, in the observations over plains, other contradictory results were obtained. In the series of papers by the Sheftel group (Sheftel and Chernyshev 1991,

Correspondence: sergey@ikir.ru

Institute of Cosmophysical Research and Radio Wave Propagation FEB RAS, Mirnaya 7, Paratunka, Kamchatskiy krai 684034, Russia
1992; Sheftel et al. 1992), the potential gradient (PG) increased relative to the background level 5 to $6 \mathrm{~h}$ before the GCR decrease was observed. This phase of field positive disturbance lasted until the Forbush effect maximum depth, then a longer negative phase of field disturbance began. A similar result was obtained in the paper by Marcz (1997): on the day of the Forbush effect maximum depth during strong geomagnetic storms, a disturbance positive phase of the electric field of approximately $2 \%$ was observed. Then, a long negative phase began with a gradual recovery of the electric field during the following 10 days. During strong geomagnetic storms, including the storm on 30 October 2003 (Nikiforova et al. 2005; Kleimenova et al. 2008) at the 'Svider' station, decreases of the potential gradient were registered. Co-occurrences in the time of their duration with the duration of splashes of riometric absorption in the subauroral zone allowed the authors to suggest that the reason for the appearance of negative values of potential gradient may be the increase of upper atmosphere conductivity caused by precipitation of energetic electrons into subauroral latitudes' (Nikiforova et al. 2005).

No less contradictory are the suggested possible mechanisms of these effects. In two papers (Markson 1981, 1982), the following hypothesis for the solar activity effect on atmospheric electricity was proposed. The essence of it is that under the effect of cosmic rays (one of the main atmosphere ionizers), the global electric circuit (GEC) conductivity changes. The GEC is a closed current system, the main generators of which, according to 
the spherical capacitor model, are tropical thunderstorms. The generator currents flow higher than it through the air resistance into the lower ionosphere and close to it through the undisturbed remote atmosphere and Earth's surface. These currents provide the charge for the Earthionosphere spherical capacitor. In the lower part of this circuit at heights up to approximately $2 \mathrm{~km}$, ground natural radio activity is the main ionizer of the atmosphere, and at heights of approximately 15 to $20 \mathrm{~km}$, the GCRs are. By penetrating into the lower stratosphere and upper troposphere, they may cause ionization that can lead to GEC current amplification. That is why, to study the solar and geomagnetic activity effects, simultaneous observations of atmospheric electricity and GCR parameters either in mountainous region or on isolated islands are needed by applying instrumentation on aircrafts or balloons higher than the exchange level of the atmosphere in which local convection and turbulence processes significantly affect the electric field strength variations. In the series of papers by Sheftel mentioned earlier, the advancing of the positive phase of the electric field strength relative to the Forbush effect and beginning at highlatitude stations is associated with the effect of solar protons, and the disturbance positive phase effect at the deepening stage is associated with the effect of the GCR hard muonic component on the atmosphere, which reaches the sea level and determines their contribution to the atmospheric conductivity at the place of electric field registration. On 28 to 31 March 2000 at the 'Borok' station, an increase of the electric field was observed during the main phase of the storm. The author suggests 'penetration of magnetospheric-ionospheric source fields into the lower atmosphere of mid-latitudes' as the effect mechanism (Anisimov 2007).

Aplin and Harrison (2014) describe three mechanisms by which a large solar storm can influence the atmospheric electricity. The first one is atmosphere ionization by solar energetic particles to the level of Earth's surface. The second type of atmospheric electrical response arises if the solar flare produces lower energy particles that enter the atmosphere but do not reach the surface. Under these circumstances, the global circuit would be modulated by decreasing the resistance of the air in the upper atmosphere, which would increase the conduction current between the ionosphere and the surface, in turn, and this would enhance the PG. The third mechanism takes place through the enhancement of lightning by solar energetic particles, which would cause increased current flow in the global circuit and, if local variations did not dominate, yield PG fluctuations.

The papers by Mikhailova et al. (2014) and Smirnov et al. (2014) illustrate the meteorological changes in the atmosphere during the solar events on 21 to 31 October 2003. Electric field conductivity and intensity showed strong dependence on air temperature and humidity. Electroconductivity increased during the 2 days before the geomagnetic storm on 29 to 30 October as a result of the solar cosmic ray (SCR) effect, and it decreased during the Forbush decrease of GCRs with the corresponding growth of the electric field intensity. An anomalous increase of air temperature and humidity during solar activity development was also detected, which led to the formation of clouds of different forms, including cumulonimbus clouds accompanied by lightning processes and showers. The coincident timing of regular meteorological process disturbances with solar flares and radiation increases in the bands of close ultraviolet, visible, and infrared spectrum sectors allows us to consider them as sources of additional energy inflow into the lower atmosphere.

\section{Methods}

Observations of geophysical fields were carried out at the Paratunka observatory of the Institute of Cosmophysical Research and Radio Wave Propagation FEB RAS, Kamchatka $\left(\lambda=158.25^{\circ} \mathrm{E} ; \phi=52.9^{\circ} \mathrm{N}\right)$. It is located on the plain, $15 \mathrm{~km}$ from the ocean, at an altitude of $50 \mathrm{~m}$ above sea level. Measurements of PG were realized by the 'Pole-2' sensor, which was developed at a branch of the Voeikov Main Geophysical Observatory (Imyanitov 1957). The Pole- 2 sensor was installed $200 \mathrm{~m}$ from the administration building at a height of $3 \mathrm{~m}$, and the area for it (radius of $12 \mathrm{~m}$ ) was cleared of trees. After digitization by a 14-bit analog-to-digital converter (ADC), the signal at the output of this fluxmeter was recorded on a personal computer (PC) hard disc with a 1-s sampling rate.

Simultaneously, air electroconductivity was measured by an 'Elektroprovodnost-2' unit that was also developed at the branch of the Main Geophysical Observatory. The unit has two inlets at heights of $3 \mathrm{~m}$ so that it can measure electroconductivity caused by positive and negative air ions separately.

\section{The principle of operation of the measuring unit for $P G$}

A unit of the electrostatic fluxmeter type is used at the observatory. The PG is transformed into electric current by a rotary electrostatic generator, in which the basis of operation involves the electrostatic induction phenomenon. The electrostatic induction current of the measured field induces an electric charge on the measuring plate. The modulator and shielding plate periodically shields the measuring plate in the electric field, and as a result, the value of induced charge periodically changes. Inflowing and outflowing charge from the plate creates current in a load circuit. The amplitude of this current is proportional to the strength of the measured electric field, modulation plate rotation frequency, and measuring plate 
square. The phase is determined by the electric field direction and by the surface of the measuring plate.

\section{The principle of operation of the measuring unit for air electroconductivity}

Measurements of air electric conductivity were carried out by the aspiration capacitor method. The air, in which electrical conductivity changes, is passed through aspiration measuring capacitors by a turbofan. Under the strength effect between capacitor plates, electric current flows on a collecting (inner) plate. This current value is proportional to the polar electrical conductivity value. By flowing through the electrometric amplifier measuring resistor, the electric current is transformed into voltage that passes to low-frequency filters and then to the output.

Field strength measurements were carried out by two channels. The first channel has a resolution of $0.25 \mathrm{~V} / \mathrm{m}$ and dynamic range of $\pm 200 \mathrm{~V} / \mathrm{m}$. The second channel has a resolution of $2.5 \mathrm{~V} / \mathrm{m}$ and dynamic range of $\pm 2000 \mathrm{~V} / \mathrm{m}$. Data from both channels were used for processing. The method used for the measurements corresponds to the manual of the Voyeikov Main Geophysical Observatory (RD 52.04.168.2001 2002).

Meteorological parameter control was carried out by digital meteorological stations WS-2000 and WS-2300. The data are transferred to the station via a radio channel at a frequency of $433 \mathrm{MHz}$. The sampling rate for meteorological data is $10 \mathrm{~min}$. The controlled parameters are as follows: wind strength, wind direction, atmospheric pressure, air temperature at 3-m height, air temperature at $25-\mathrm{m}$ height, air humidity, precipitation (in the summer time), solar radiation intensity, and solar energy flux density.

Measurements of geomagnetic field variations were carried out by a fluxgate magnetometer FRG-601G with a 1-s sampling rate and 0.01-nT error.

\section{Results}

The minimum of the 23-d cycle of solar activity was in December 2008. The period 2006 to 2010 was characterized by a small number of magnetic storms. On 3 April 2010, an X-ray flare of the B7.4 class occurred on the Sun, which led to coronal filament ejection. Its intensity was not big, but it was quite long (more than $7 \mathrm{~h}$ ). On 5 April 2010 at 8:27 UT, the beginning of a magnetic storm with a sudden commencement was registered. Figure 1 shows the 1-min averaged data for the geophysical field measurements at the Paratunka site. Figure 1a shows the graph of the geomagnetic field $H$-component on 5 to 6 April. The $K$-index of the storm was 7. A previous storm of the same class occurred on 11 October 2008, i.e., the analyzed event was the strongest storm for the previous year and a half.
Magnetic storm effects on the near-ground electric state may be divided into three stages. The background level of PG before the storm was about $25 \mathrm{~V} / \mathrm{m}$ (Figure 1b). Field low level is determined by the seasonal variation of high amplitudes from $60 \pm 40$ to $120 \pm 60 \mathrm{~V} / \mathrm{m}$. For this month at this observatory, the field low level was characteristic, i.e., there was nothing unusual about it. For example, the paper by Siingh et al. (2013) showed PG diurnal variation at different sites during various seasons in the range from 48 to $82 \mathrm{~V} / \mathrm{m}$ andto from 130 to $185 \mathrm{~V} / \mathrm{m}$. The first stage from 4:25 untilto 8:27 UT was characterized by an increase of the electric field level up to $50 \mathrm{~V} / \mathrm{m}$. Simultaneously, the air electroconductivity experienced a twofold decrease (Figure 1c). The cause for such an increase in the PG might have been the sharp decrease of the GCR penetration level to Earth's surface. Two factors support this interpretation. First, conduction current density variations during this period were not as significant as during the following time interval and did not change considerably (Figure $2 \mathrm{~b}$ ). This value was received by an indirect method from the calculation $j=\operatorname{Ez} \times\left(\lambda_{+}+\lambda_{-}\right)$, where $\mathrm{Ez}$ is the electric field strength, and $\lambda_{+}$and $\lambda_{-}$represent the electroconductivity of the air caused by positive and negative ions, respectively. Second, air electroconductivity sharply decreased during this period (Figure 1c). The GCRs together with radon are air ionizers. A decrease in the GCR ionization effect leads to a decrease in the electroconductivity (Figure 1c) and, consequently, to an increase of PG (Figure 1b). There is no station for cosmic ray measurements in Kamchatka and nearby regions. Figure 3 shows the data for the proton flux according to GOES-15 data and cosmic ray indices of Moscow. The data were received from the National Geophysical Data Center site: spidr.hgdc.noaa.gov. It is possible that ionization processes in the near-ground layer of mid-latitudes are so complicated and nonlinear that they had such an effect in Kamchatka.

During the second stage in the interval from about 8:27 to 12:00 UT, a sharp increase in the electric field and then a decrease occurred. This coincided in time with sharp oscillations of the geomagnetic field $H$-component, and a similar behavior was observed in the conduction current density. Such disturbances are likely to have an induction nature. Figure $2 \mathrm{c}$ shows the graph of magnetic field measurements $\Delta H_{i}=H_{i+1}-H_{i}$, where $H_{i}$ is the measurement series of the magnetic field $H$-component with 1 -min averaging. Figure $2 \mathrm{~b}$ shows the conduction current density. It is clear from Figure 2 that the storm with sudden commencement significantly changed the current system in the near-ground layer. But during the following stages, the magnetic disturbance effect on the current system was weak.

At the third stage from about 12:00 UT on 5 April to 1:30 UT on 6 April, an electric field increase occurred, 


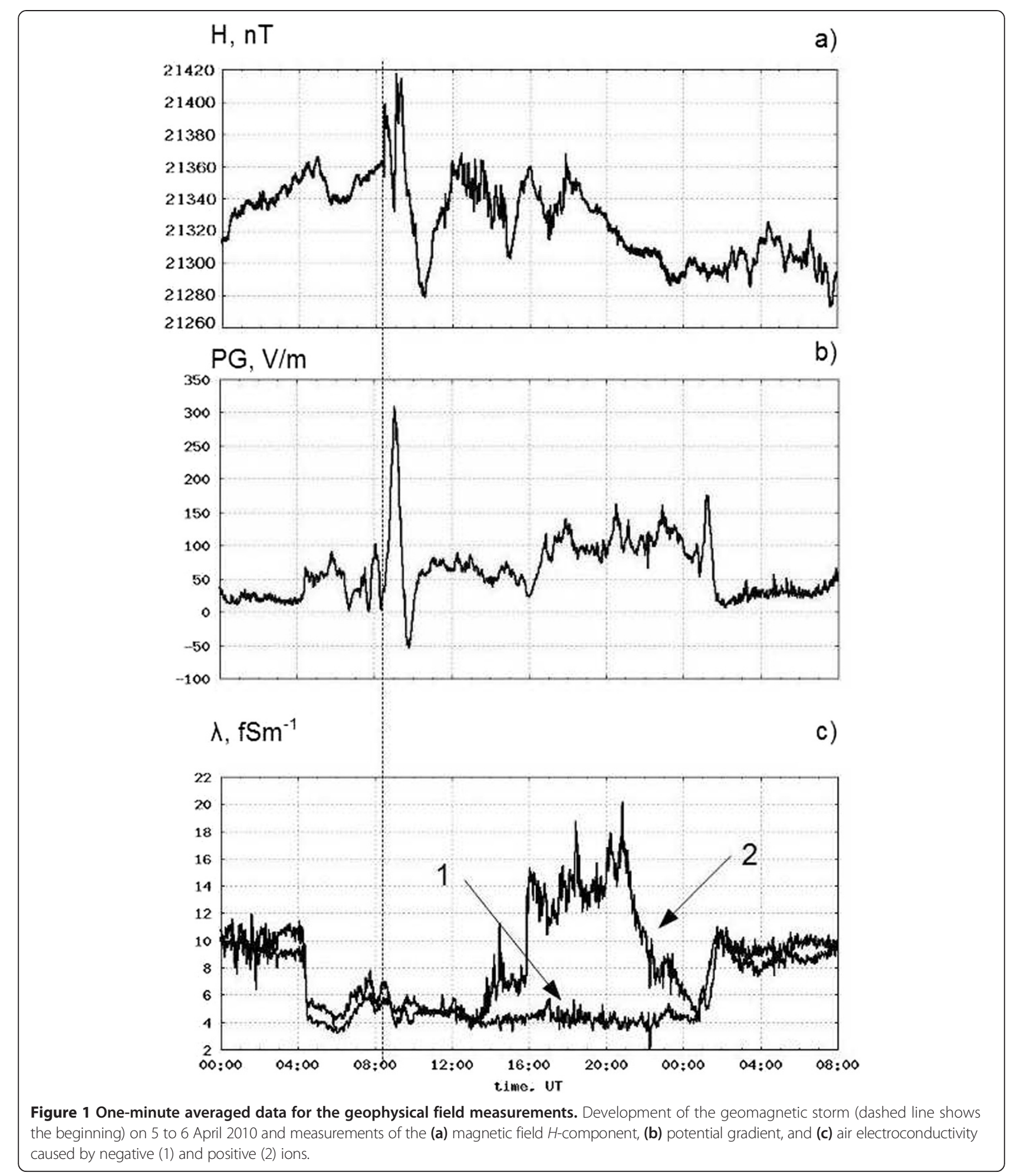

which was associated with an increase of the unipolarity coefficient (Figure 2c). The unipolarity coefficient $K=\lambda_{+} / \lambda_{-}$takes into account the ion concentration and ion mobility. Observations at the Paratunka observatory show that the rain effect causes a decrease in the unipolarity coefficient, and the snowfall effect causes an increase. The vertical current density during this time exceeded the level of the second stage (Figure 2b). 

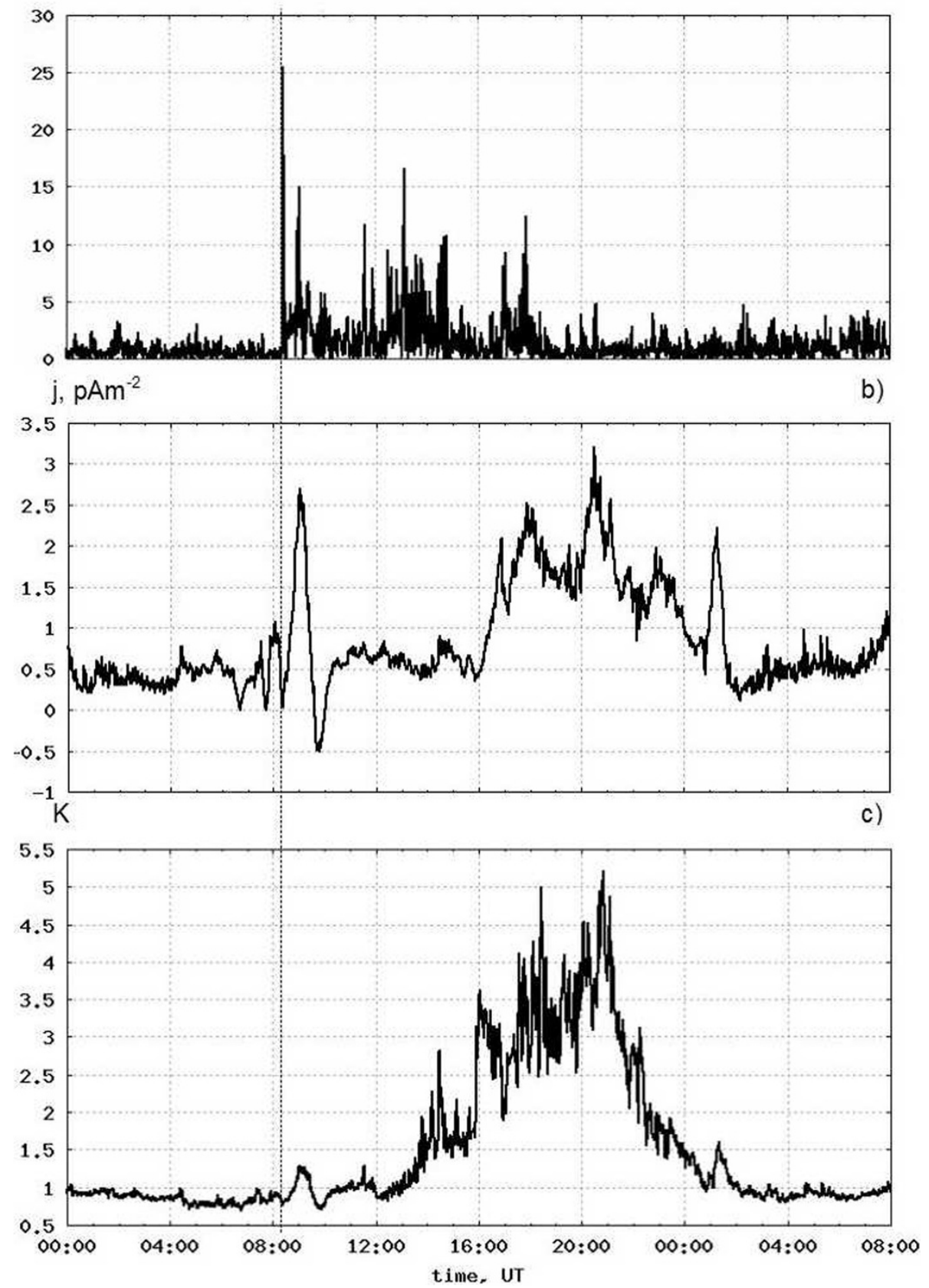

Figure 2 Development of the geomagnetic storm and graphs of measurements. Development of the geomagnetic storm (dashed line

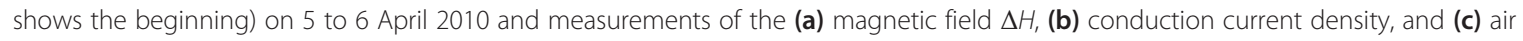
unipolarity $(\lambda+/ \lambda-)$.

\section{Discussion}

Investigations of magnetic storm effects on the electric state of the near-ground air have been discussed in many papers. Unfortunately, the results of these investigations are contradictory. This may be the result of not only the peculiarities of physical processes in the near-ground atmosphere for separate magnetic storms but also the choice of the location for field registration. No less contradictory are the possible mechanisms of these effects. An overview of these mechanisms was presented in the paper by Smirnov et al. (2013). In this study, three effects were traced during the development of a storm. The first one was associated with a decrease in the air electroconductivity (Figure 1c). Such a decrease might have been driven 


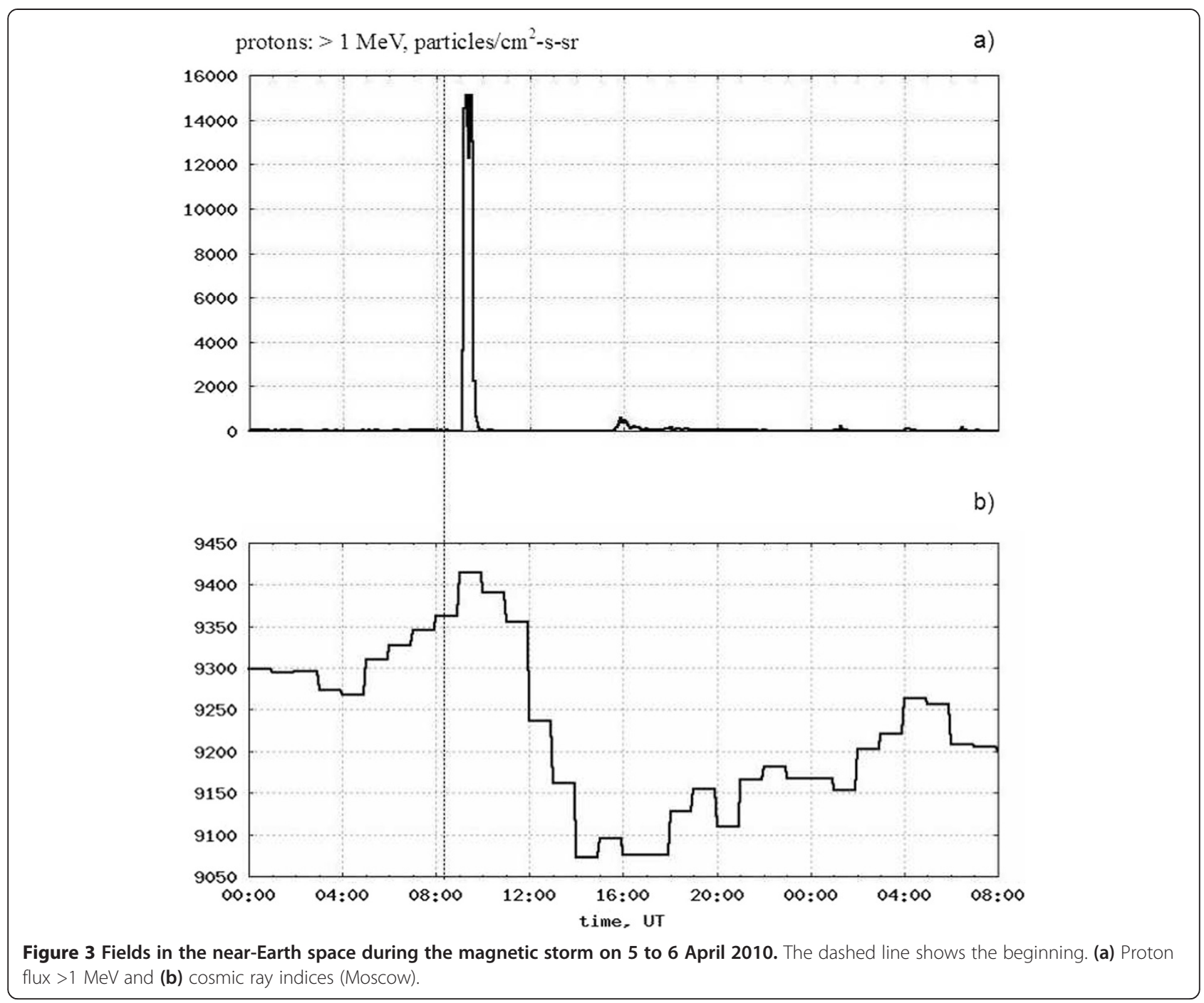

by the 'turn off' of one of the ionizers of air molecules. Ionizers at this level are radon and GCRs. The seismic state during this time was calm, which means that there were no significant deformation processes that could have led to a sharp increase of radon emanation. Hence, the electroconductivity decrease may have been associated with a decrease in the GCR flux.

The second effect appeared as current sharp changes at the initial stages of the storm. Evidently, this effect was associated with induction phenomena of electromagnetic processes. It is now quite clear what these processes are. One of the mechanisms was suggested in the paper by Nicoll and Harrison (2014). That paper proved experimentally that solar energetic particles occasionally contribute additional atmospheric ionization beyond that arising from the usual GCR background. That must have led to the electric field change on Earth's surface, but it was not displayed on neutron monitor indications.
The third effect involved an increase of the unipolarity coefficient. Such excess could have been caused by very heavy snowfall in the observation region. Since the draft indicator did not register any precipitation, it is possible that precipitation in the form of small snowflakes had a weak effect. Wintertime observations at the Paratunka observatory show that the snowfall effect causes an increase unipolarity coefficient. Meteorological processes during the storm may have amplified this effect.

The behavior of meteorological parameters during the solar flare and magnetic storm from 11 to 13 April 2010 is presented in Figure 4. The curve in Figure 4a shows the Ap index of geomagnetic activity, Figure $4 \mathrm{~b}$ is the air temperature in the near-ground layer, Figure $4 \mathrm{c}$ is the air humidity, Figure $4 \mathrm{~d}$ is the Sun energy flux density, Figure $4 \mathrm{e}$ is the atmospheric pressure, and Figure $4 \mathrm{f}$ is the wind strength. On 2 April, the weather was bad; there was precipitation that was confirmed by humidity 

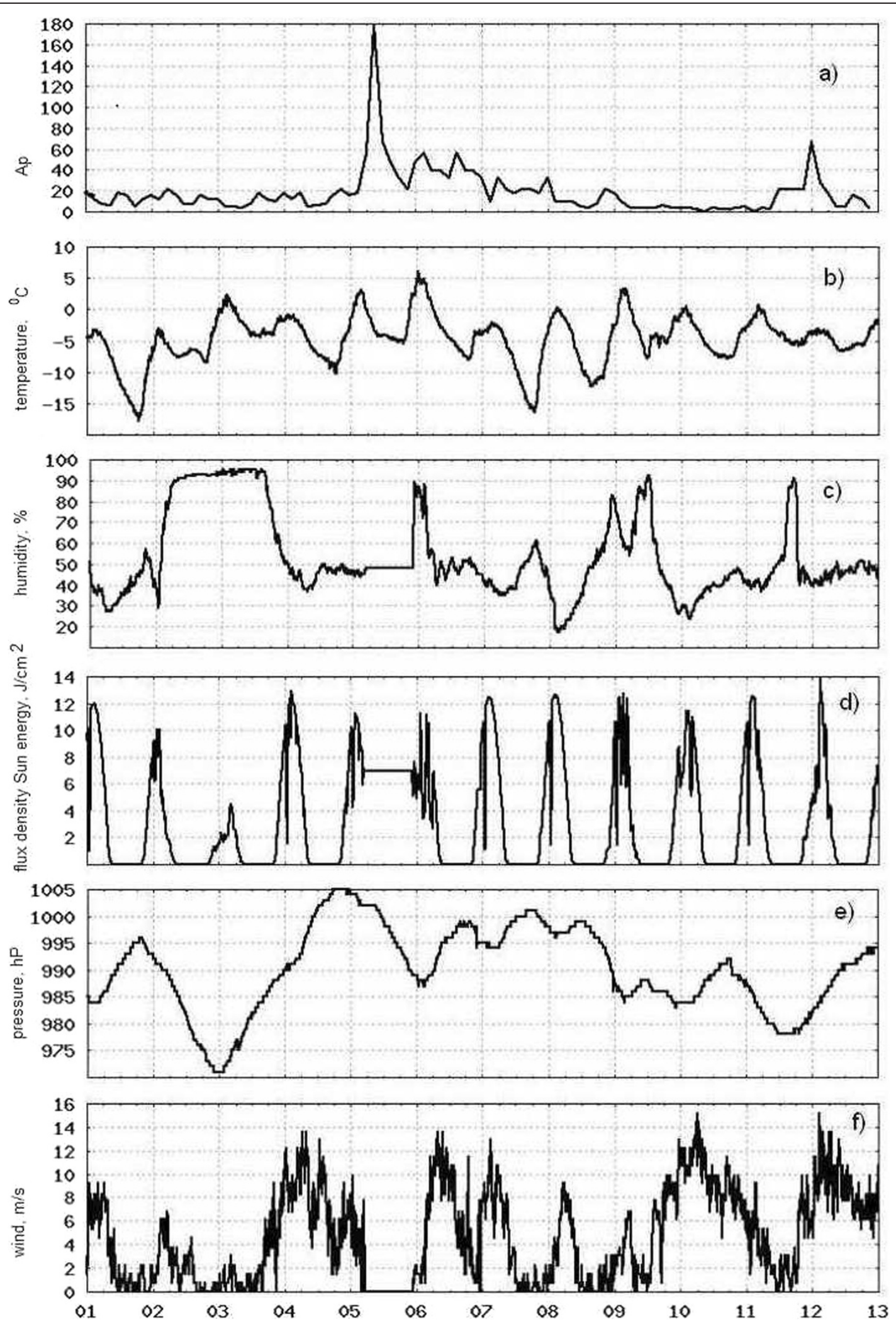

Figure 4 Behavior of meteorological parameters during the magnetic storm. The curves show the (a) Ap index of geomagnetic activity, (b) air temperature in the near-ground layer, (c) air humidity, (d) Sun energy flux density, (e) atmospheric pressure, and (f) wind strength. 
curves (Figure 4c) and by the Sun energy flux (Figure 4d). Horizontal lines on 5 April denote data unavailability. On the second day after the flare, on 4 April, the weather was fine, clear, and sunny. The nighttime temperature increase on 2 April may be explained by bad weather conditions, but on the following days up to 7 April, this increase was possibly associated with solar flare effects. A similar effect was observed during magnetic storms on November 2004 (Smirnov et al. 2013). In light (Danilov and Lastovika 2001) of the possible mechanisms for geomagnetic storm effects on the troposphere, it is a dynamic mechanism associated with the increase of acoustic-gravitation atmospheric waves at the planetary scale; it is an electric mechanism associated with the global electric circuit and cosmic ray penetration into the atmosphere; it is an optical mechanism associated with atmosphere transparency changes and chemical contents in the stratosphere under the influence of short-wave radiation of the Sun and GCRs. The change of just nighttime temperatures is indicative of the processes slowing heat loss.

\section{Conclusions}

In this study, the influence of a geomagnetic storm on the near-ground air electric state was investigated. The following effects were observed:

1. Air electroconductivity decreased by a factor of 2 . This effect began $4 \mathrm{~h}$ before the sudden commencement of the magnetic storm and lasted for about $20 \mathrm{~h}$.

2. Large oscillations of the potential gradient with amplitudes up to $300 \mathrm{~V} / \mathrm{m}$ were observed, and they coincided with the beginning of the magnetic storm. Such disturbances were, possibly, caused by induction processes. The duration of this process was about $2 \mathrm{~h}$.

3. Field level increase up to the level of $150 \mathrm{~V} / \mathrm{m}$. This increase was associated with ion content change in the near-ground atmosphere. The ion unipolarity coefficient increase in the atmosphere showed that positive ion concentration and mobility significantly exceeded those of negative ions. The conduction current also increased. This effect occurred $8 \mathrm{~h}$ after the magnetic storm commencement and lasted for about $13 \mathrm{~h}$.

\section{Abbreviations}

GCR: galactic cosmic ray; GEC: global electric circuit; PG: potential gradient; SCR: solar cosmic ray.

\section{Competing interests}

The author declares that he has no competing interests.

\section{References}

Anisimov SV (2007) Geophysical aspects of investigation of the global electric circuit. In: Paper presented at the 6th Russian Conference on Atmospheric Electricity, Nizhniy Novgorod, 1-7 October 2007

Aplin KL, Harrison RG (2014) Atmospheric electric fields during the Carrington flare. Astron Geophys 55(5):5.32-5.37, doi:10.1093/astrogeo/atu218

Apsen AG, Kanonidi KD, Chernyshova SP, Chetaev DN, Sheftel VM (1988) Magnetospheric effects in atmospheric electricity. Nauka, Moscow

Danilov AD, Lastovika J (2001) Effects of geomagnetic storms on the ionosphere and atmosphere. Int J Geomagn Aeron 2(3):209-224

Imyanitov IM (1957) Instrumentation and methods to investigate atmospheric electricity. Gostechizdat, Moscow

Kleimenova NG, Kozyreva OV, Michnowski S, Kubicki M (2008) Effect of magnetic storms in variations in the atmospheric electric field at midlatitudes. Geomagn Aeron 48(5):622-630, doi:10.1134/S0016793208050071

Marcz F (1997) Short-term changes in atmospheric electricity associated with Forbush decreases. J Atmos Solar-Terrestrial Phys 59(9):975-982

Markson R (1981) Modulation of the Earth's electric field by cosmic radiation. Nature 291:304-308

Markson R (1982) Atmospheric electricity and the problems of relation between the solar activity and weather. In: Mak-Korman B, Seliga T (eds) Solar-terrestrial relations, weather and climate. Mir, Moscow, pp 242-264

Mikhailova GA, Mikhailov YM, Kapustina OV, Druzhin Gl, Smirnov SE (2009) Power spectra of thermal tidal and planetary waves in the near-earth atmosphere and in the ionospheric D region at Kamchatka. Geomagn Aeron 49(5):610-623, doi:10.1134/S0016793209050089

Mikhailova GA, Kapustina OV, Smirnov SE (2014) Effects of solar and geomagnetic activities in variations of power spectra of electrical and meteorological parameters in the near-Earth atmosphere in Kamchatka during October 2003 solar events. Geomagn Aeron 54(5):645-654, doi:10.1134/S0016793214050119

Nicoll KA, Harrison RG (2014) Detection of lower tropospheric responses to solar energetic particles at mid-latitudes. Phys Rev Lett 112:225001

Nikiforova NN, Kleimenova NG, Kozyreva OV, Kubitski M, Michnowski S (2005) Unusual variations in the atmospheric electric field during the main phase of the strong magnetic storm of October 30, 2003, at Swider Polish midlatitude observatory. Geomagn Aeron 45(1):140-144

RD 52.04.168.2001 (2002) Manuals on observations of the electric field. Gidrometeoizdat, St. Petersburg

Sheftel VM, Chernyshev AK (1991) Solar flare effects in atmospheric electricity at high and mid latitudes. Geomagn Aeron 31(3):500-505

Sheftel VM, Chernyshev AK (1992) Atmospheric electricity response to an isolated flare and to series of flares. Geomagn Aeron 32(1):111-117

Sheftel VM, Bandilet OM, Chernyshev AK (1992) Effects of planetary magnetic storms in the atmospheric electric field near the earth's surface. Geomagn Aeron 32(1):186-188

Siingh D, Singh RP, Gopalakrishnan V, Selvaraj C, Panneerselvam CP (2013) Fair-weather atmospheric electricity study at Maitri (Antarctica). Earth Planets Space 65(12):1541-1553, doi:10.5047/eps.2013.09.011

Smirnov SE, Mikhailova GA, Kapustina OV (2013) Variations in the quasi-static electric field in the near-Earth's atmosphere during geomagnetic storms in November 2004. Geomagn Aeron 53(4):502-514, doi:10.1134/ S0016793213040130

Smirnov SE, Mikhailova GA, Kapustina OV (2014) Variations in electric and meteorological parameters in the near-Earth's atmosphere at Kamchatka during the solar events in October 2003. Geomagn Aeron 54(2):240-247, doi:10.1134/S0016793214020182

doi:10.1186/s40623-014-0154-2

Cite this article as: Smirnov: Reaction of electric and meteorological states of the near-ground atmosphere during a geomagnetic storm on 5 April 2010. Earth, Planets and Space 2014 66:154. 